\title{
Theoretical and Experimental Studies of Tubular Valve Dynamics
}

\author{
Minvydas Ragulskis and Arvydas Palevicius \\ Kaunas University of Technology, P.O. Box 1300, LT-3028 Kaunas, Lithuania
}

(Received 27 October 2000; accepted 31 August 2001)

Construction of a novel vibratory valve and its design optimisation is presented in this paper. The principle of the system's operation is based on the effect of the dynamic positioning of a steel ball in a vibrating tube. A theoretical analysis of the stability of this non-linear system together with an experimental study of the operating valve forms the basis of this study. Laser holographic interferometry was used for the identification and optimisation of the regimes of operation of the system.

\section{INTRODUCTION}

Liquid material controlled dosing and spraying has different applications - from medicine to agriculture. ${ }^{1,2}$ Specific interest exists for elastic catheter pipe type dosing equipment. Complex dynamic processes that take place in such systems are analysed in references ${ }^{3,4}$. A method to study the dynamic characteristics of a tube under the influence of an internal flow is presented in reference ${ }^{3}$. Galerkin's method in conjunction with the method of multiple scales is employed for obtaining the stability of the tube vibration. According to the results, instability can occur under certain conditions of resonance. It is shown in reference ${ }^{4}$ that fluid-elastic effects which are responsible for fluid-elastic instabilities may be directly measured through the analysis of the vibrating motion of a system under flow. Piezoelectric actuators are used to increase the vibratory level when buffeting forces which excite tube vibration are low, and to improve the measurement of fluid-elastic forces.

Application of piezoelectric actuators for the generation of standing waves in the outlet pipe can produce effects which can be used for the control of the dosing process. The motion of fluid-suspended particles and fibres in a standing wave field is analysed in references ${ }^{5,6}$. The dynamics of micron aerosol particles and their agglomeration under standing wave conditions is analysed in reference ${ }^{7}$. Coupling of the dynamic properties of a vibrating tube with the dynamic behaviour of a steel ball inside the tube can help producing a new type of smart doser of liquid material which can be effectively controlled by piezoelectric actuators. The unique feature of the tubular vibratory valve consists in the fact that the sealing surface of the seat is faces towards the intake duct and is located at the node of the second natural mode of transverse vibration of the elastic pipe. The vibratory valve for controlling liquid flow (Fig. 1) operates in the following fashion.

The liquid that is fed into the intake duct (4) by the force of the flow which depends on the pressure in the system brings the locking ball (6) into sealing contact with the seat (7). The valve closes and the flow of liquid through the outlet duct is interrupted. When the driving generator (6) sends control signals (the frequency of which corresponds to the second natural frequency of transverse vibration of the pipe) to the vibrator (2), the latter excites transverse vibrations in the pipe. Since the frequency of the exciting oscillations of the vibrator (2) corresponds to the second natural frequency of transverse vibration of the pipe, it initiates transverse vibration of the second natural mode at the resonance frequency. As a result, the locking element (6) overcomes the force of the flow and shifts to the point where the transverse vibration of the pipe reaches its maximum amplitude: the seat valve (7) opens and the liquid flows through the outlet duct (5). Figure 1 presents a design diagram of a vibrator valve for controlling liquid flow. It also shows the second natural mode of transverse vibration of the pipe and the location of the locking element with respect to the seat when the valve is open.

\section{DYNAMICS OF A STEEL BALL INSIDE A VIBRATING TUBE}

Motion of a small ball inside a tube performing transverse vibrations may be approximated by the non-dimensional differential equation of motion describing the dynamics of a mass particle on an oscillating profile with an obligatory condition of contact with the surface: $:^{3,4}$

$$
\begin{gathered}
\left(1+\left(\frac{\partial \zeta}{\partial x}\right)^{2}\right) m \ddot{x}+\frac{\partial^{2} \zeta}{\partial x^{2}} \frac{\partial \zeta}{\partial x} m(\dot{x})^{2}+2 \frac{\partial^{2} \zeta}{\partial x \partial t} \frac{\partial \zeta}{\partial x} m \dot{x} \\
+h\left(1+\left(\frac{\partial \zeta}{\partial x}\right)^{2}\right) \dot{x}+\frac{\partial^{2} \zeta}{\partial t^{2}} \frac{\partial \zeta}{\partial x} m+\frac{\partial \zeta}{\partial x} m g=F
\end{gathered}
$$

where $\zeta=\zeta(x, t)$ is the mode shape of the tube; $x, \dot{x}, \ddot{x}$ are projections of the displacement, velocity and acceleration of the ball on the horizontal axis; $t$ is the time; $m$ is the mass of the ball; $h$ is the coefficient of viscous friction between the ball and the surface of the profile; $g$ is the acceleration of gravity; and $F$ is the pressure force of the liquid.

Naturally, Eq. (1) is based on the assumption that the area of the cross section of the tube is small, the fluid flow is laminar, and the amplitude of the pressure of the liquid in the tube is not aligned with the amplitude of the elastic transverse vibrations. Also it is assumed that the mass of the locking ball is sufficient enough to perform the vibration-induced motion in the liquid, but not big enough to alter the shape of the tube's vibration due to its relocation. 